INTERDISCIPLINARIA ARCHAEOLOGICA NATURAL SCIENCES IN ARCHAEOLOGY

\title{
Environment and Economy of the Early Medieval Settlement in Žatec
}

\author{
Petr Kočára ${ }^{\mathrm{a}}$ Petr Čech ${ }^{\mathrm{b}}$, Radka Kozákováb ${ }^{*}$, Romana Kočárováa \\ ${ }^{a}$ Department of Archaeology, Faculty of Arts, West Bohemian University, Sedláčkova 268/38, 30614 Pilsen, Czech Republic \\ ${ }^{b}$ Institute of Archaeology of Academy of Sciences of the Czech Republic, Letenská 123/4, 11800 Prague, Czech Republic
}

\section{ARTICLE INFO}

\section{Article history:}

Received: 6 December 2010

Accepted: 18 February 2011

\section{Key words:}

Žatec,

Early Medieval Period

plant macroremains

agriculture, cereals

pollen analysis

charcoal analysis

three field system

\begin{abstract}
A BSTRACT
Long term archaeological research of the Early Medieval agglomeration at Žatec was combined with archaeobotanical analyses of urban layers. Macroremains, pollen and charcoal samples were taken mostly from sunken settlement objects and moats that were a part of the urban fortification. Analysed sediments were dated according to a detailed pottery chronology. The main aim of the research was to detect important changes in cereal crop production that coincided with the development of the town as evidenced in the archaeological record. Pollen and charcoal data were used to reconstruct the natural environment of the town and its vicinity. Archaeological excavations showed intensified development of the town during the $11^{\text {th }}$ century, a transition supported by the macroremains analysis. Urban layers from the $9^{\text {th }}$ and $10^{\text {th }}$ centuries contain large numbers of naked wheat macroremains, while from the $11^{\text {th }}$ century there is marked increase in the abundance of rye and weeds of winter crops. Such changes can point to a different, more intensive, system of farming that was similar or identical to a three field system. Pollen spectra available for the $9^{\text {th }}$ and $10^{\text {th }}$ century evidence extensive deforestation around Žatec. Synanthropic and cultivated taxa were dominant among herbs, typical for medieval towns, and only a small proportion of pollen spectra included the grassland taxa indicative of a warm and dry climate. According to the analysis of charcoals and non carbonized wood, Pinus and Quercus were the prevailing tree taxa. Increasing finds of fir, beech, spruce and yew charcoal during the $11^{\text {th }}$ and $12^{\text {th }}$ centuries is likely connected with wood imported during the development of iron-working. In any case the main outcome of the research in Žatec is the relatively early dating, to the $11^{\text {th }}$ century, of the agricultural shift that is generally supposed to be connected with the High Medieval transformation of the Czech state during the $13^{\text {th }}$ century.
\end{abstract}

\section{Introduction}

The form and development of the Early Medieval agglomeration at Žatec have been recently outlined using a geomorphologic description of the location, agglomeration space zoning and information derived from relative and absolute pottery chronologies (Čech 2008a, 36-60). That study was devoted to completing an outline of the pottery chronology based on select and specific terrain situations (Čech 2008b, 91-102). As part of the project, samples of anthropogenic sediments have been taken during planned and salvage excavations since 1999. These were collected for the purpose of obtaining fossil organic material for analyses related to the economy of

"Corresponding author. E-mail: R.Kozakova@seznam.cz the Early Medieval period, with an emphasis on changes in agriculture. In addition, the project is focused on ecological conditions in the agglomeration, their development and changes, based on archaeobotanical and pollen analyses. Over a decade more than one hundred samples were gathered from the locality, dating back to the middle and late phases of the Early Medieval period ( $9^{\text {th }}$ to early $13^{\text {th }}$ century).

\section{Methods}

The first anthropogenic sediment samples were taken in 1999. Emphasis was given to the so-called primary filling of recessed objects - those layers that created the filling of the object itself, not the layers that recessed into the filling later on. Sampling continued in 2000 and the largest sets were 
obtained in 2004-2006 from excavations in the area of a hot water pipeline that divides Žatec north to south. Sample units consisted of one bucket (approximately 10 litres) of sediment. The aim was to collect and compare material from the area of the castle and the surrounding settlement. Samples from the surrounding settlement are less numerous, but continue to be collected in those areas.

Samples were floated and wetsieved on a regular basis, mostly outdoors, with a smaller portion treated in the laboratory using an identical device (Jones 1991; Van der Veen 1984). A modified version of an "Ankara" wetsieve line with mesh diameter to $0.25 \mathrm{~mm}$ (Pearsall 1989) was used. Floating fractions were dried at room temperature. Plant macroremains were selected and separated under a stereoscopic microscope with up to 90x magnification. Palaeobotanical material was identified according to the collection of plant diaspores at the Department of Archaeology, Faculty of Arts, West Bohemian University, Pilsen, Czech Republic. Literature for identifying plant macroremains was also consulted (Anderberg 1991; Beijerinck 1947; Berggren 1969, 1981; Bertsch 1941; Katz et al. 1965; Schermann 1967).

A chronological division into 6 time periods based on detailed archaeological typology (Čech 2008a) was modified, for the purposes of archaeobotanical data analysis, into 4 time periods:

- $9^{\text {th }}$ century (beginning $\left.10^{\text {th }}\right)=$ Žatec B, B-C and B-D;

- $10^{\text {th }}$ century $=\mathrm{D}$ and $\mathrm{C}-\mathrm{D}$;

- $11^{\text {th }}$ century $=\mathrm{E}$;

- $12^{\text {th }}$ century $=$ pottery of $\mathrm{F}$ and $\mathrm{F}-\mathrm{G}$.

The wild plants identified were further divided for analysis on the basis of recent ecological requirements into several groups:

1. Weeds of winter crops (including those cultivated on both basic-poor and basic-rich soils) - Agrostemma githago, Anthemis arvensis, Avena sp., Bromus arvensis, Vicia sp., Scleranthus annuus, Vicia tetrasperma/hirsuta, Adonis aestivalis, Bupleurum rotundifolium, Stachys arvensis/annua, Camelina microcarpa, Melampyrum arvense.

2. Weeds of summer crops (including weeds of pulses and millet) - Atriplex patula, Chenopodium album, Chenopodium sp., Chenopodium hybridum, Chenopodium polyspermum, Chenopodium murale, Polygonum aviculare agg., Persicaria lapathifolia, Lamium sp., Fallopia convolvulus, Fumaria officinalis, Galeopsis tetrahit type, Lapsana communis, Malva sp., Sagina procumbens, Sinapis arvensis, Thlaspi arvense, Viola arvensis agg. Echinochloa cruss-gali, Setaria pumila, Setaria viridis/verticillata, Galium spurium, Neslia paniculata.

3. Plant species of various grassland communities Agrimonia eupatoria, Agrostis sp., Carex sp., Carex spicata/muricata, Centaurea jacea, Leontodon autumnalis, Securigera varia, Stipa sp., Trifolium pratense, Trifolium repens, Medicago sp., Plantago lanceolata, Ranunculus acris.
4. Plant species of fallow and ruderalised lands-Agropyron repens, Convolvulus arvensis, Rumex crispus, Rumex sece Rumex, Carex hirta, Ranunculus repens, Bromus sterilis, Galeopsis angustifolia, Galium aparine.

\section{Taphonomy of the plant macroremains}

Plant macroremains were isolated by wetsieving from mainly dry archaeological layers. The majority were comprised of 8,220 carbonized plant macroremains, 4,373 non- carbonized macroremains and 2,448 mineralized macroremains.

The state of preservation of plantmacroremains is a suitable indicator of deposition conditions within the sampled layers. Mineralized macroremains indicate unsuitable periodically dry conditions, whereas non-carbonized macroremains are only well-preserved in constantly anaerobic (oxygenfree) conditions. Carbonized macroremains, formed by burning, are not directly affected by deposition conditions since they are resistant to unfavourable conditions present in sediments. When studying these groups of plant macroremains, defined in accordance with the manner of preservation in individual chronological time periods, it is clear that individual time periods significantly differ with respect to the manner of preservation of plant remains. Thus, the conditions of deposition during individual time periods probably significantly differed as well. Layers from the oldest period contain predominantly mineralised plant remains $(71 \%)$, whereas non- carbonized remains account only for $11 \%$ out of the total number of macroremains (plant remains from this period came mostly from dry aerated layers). Over time the number of non-carbonized plant remains increases, up to $93 \%$ in the $12^{\text {th }}$ century, with only $1 \%$ mineralized remains (e.g. the preservation conditions tend towards being constantly anaerobic). See Table 1 and Figure 1.

It is clear that time is the main factor influencing the conditions of macroremain preservation. As time progresses the organic elements of anthropogenic sediments that hold moisture gradually decay. Moisture loss results in a gradual loss of the anaerobic environment and the creation of conditions suitable for mineralization of seeds with a relatively solid coat that resists biological decomposition. Another factor is the subsoil (loess) that contains silicates and calcareous compounds that cause mineralization of the seed hulls.

In order to compare the individual periods we must focus on carbonized plant remains. Whereas mineralized and to some extent non-carbonized macroremains show signs of selective preservation (e.g. considerably high concentration of species with resistant seeds or fruits), the carbonized remains are the least affected (carbonization completely conserves the plant material during the process of burning). Therefore, the set of carbonized remains was used as the reference for studying the palaeo-ecological conditions of the locality and changes over time. 
Figure 1. Žatec - ratio of carbonized, noncarbonized and mineralized plant remains, by century.

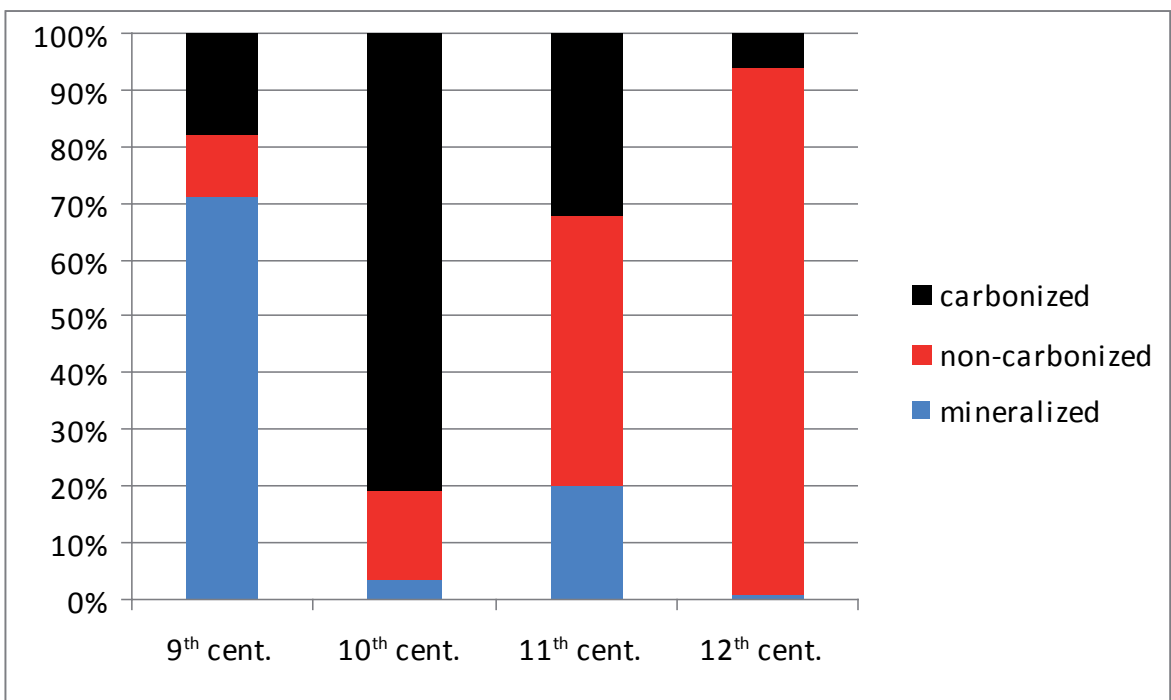

Table 1. Results of pollen analysis confronted with the findings of plant macroremain analysis. Pollen types were defined according to Reille (1992), Beug (2004) and Punt (1980)

\begin{tabular}{|c|c|c|c|c|c|c|c|c|c|}
\hline Layer number & 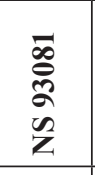 & 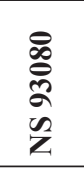 & 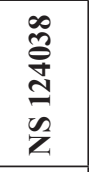 & 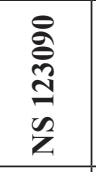 & 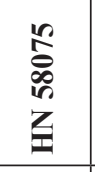 & 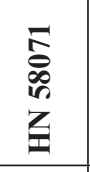 & 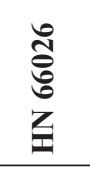 & & \\
\hline Archaeological dating & 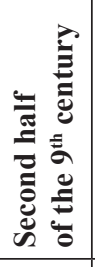 & 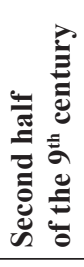 & 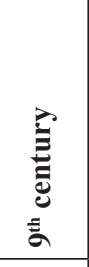 & 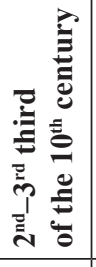 & 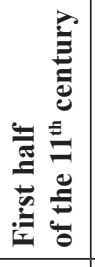 & 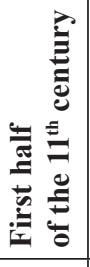 & 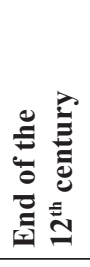 & & \\
\hline $\begin{array}{c}\text { Archaeological } \\
\text { context }\end{array}$ & 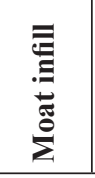 & 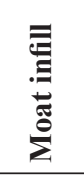 & 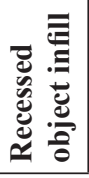 & 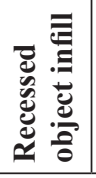 & 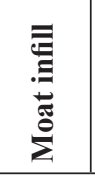 & 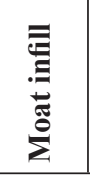 & 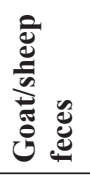 & & \\
\hline Pollen type & \multicolumn{7}{|c|}{$\%$ ratio in pollen spectrum } & Macroremain analysis & Prevailing type of biotope \\
\hline Abies alba & 0.2 & 0.3 & & & 0.5 & 1.8 & & & forests, cultural forests \\
\hline Alnus & 0.4 & 0.1 & 0.5 & 0.2 & & & & & floodplain forests, carrs \\
\hline Betula & 0.1 & 0.4 & 2.1 & & 0.2 & & & & forest clearings \\
\hline Carpinus betulus & & & & & & 0.7 & & & forests, cultural forests \\
\hline Corylus & & & & & 0.5 & & 1.7 & & $\begin{array}{l}\text { cultural forests, forest } \\
\text { margins, shrubs }\end{array}$ \\
\hline Fagus sylvatica & & & 0.2 & & & & 1.0 & & forests in higher elevations \\
\hline Picea & & 0.1 & & 0.7 & & 0.3 & & & forests in higher elevations \\
\hline Pinus & 1.3 & 1.6 & 0.5 & 0.9 & 0.9 & 0.9 & 0.2 & & dry forests, cultural forests \\
\hline Quercus & 0.1 & 0.1 & 1.0 & 0.4 & & & 0.2 & & $\begin{array}{l}\text { lowland forests, floodplain } \\
\text { forests }\end{array}$ \\
\hline Tilia & & 0.1 & 0.2 & & & & & & lowland forests \\
\hline Prunus type & & 0.3 & & & & & & $\begin{array}{l}\text { Malus/Pyrus, Prunus } \\
\text { avium/cerasus, Prunus } \\
\text { insititia }\end{array}$ & orchards, shrubs \\
\hline Sambucus & & & & & & & 0.6 & & shrubs, ruderal places \\
\hline Adonis aestivalis type & & 0.8 & & & & 0.7 & & Adonis aestivalis & fields, fallow land \\
\hline Agrostemma githago & 0.1 & & 0.2 & 0.2 & & & & Agrostemma githago & cereal fields \\
\hline
\end{tabular}


Table 1. Results of pollen analysis confronted with the findings of plant macroremain analysis. Pollen types were defined according to Reille (1992), Beug (2004) and Punt (1980) (continued).

\begin{tabular}{|c|c|c|c|c|c|c|c|c|c|}
\hline Layer number & 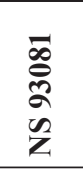 & 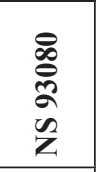 & 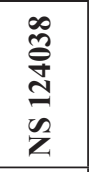 & 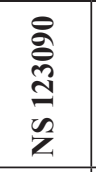 & 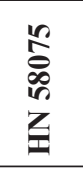 & 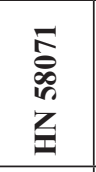 & $\begin{array}{l}\text { đัँ } \\
\text { : } \\
\text { ż } \\
\text { z }\end{array}$ & & \\
\hline Archaeological dating & 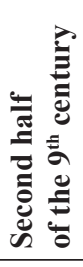 & 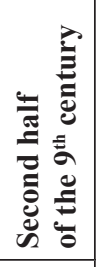 & 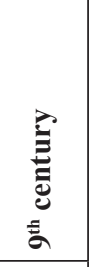 & 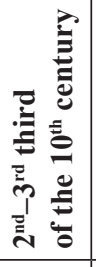 & 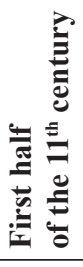 & 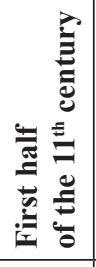 & 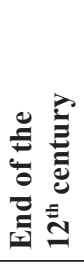 & & \\
\hline $\begin{array}{l}\text { Archaeological } \\
\text { context }\end{array}$ & 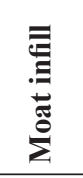 & 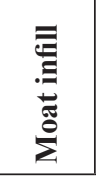 & 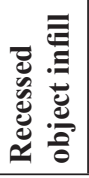 & 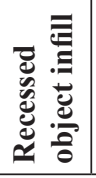 & 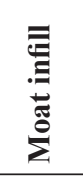 & 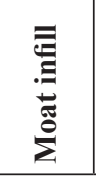 & 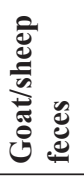 & & \\
\hline Pollen type & \multicolumn{7}{|c|}{$\%$ ratio in pollen spectrum } & Macroremain analysis & Prevailing type of biotope \\
\hline Alchemilla & 1.7 & 1.3 & 0.7 & 0.7 & 0.5 & 0.5 & & & $\begin{array}{l}\text { path margins, pastures, } \\
\text { ruderalized grasslands, } \\
\text { moats }\end{array}$ \\
\hline Anthericum & & 0.1 & & & & & & & dry grasslands \\
\hline Apiaceae & 1.5 & 1.3 & 1.7 & 0.4 & & & 1.4 & & \\
\hline Arctium & & & & & & & 0.4 & & ruderal places \\
\hline Artemisia & 4.8 & 4.2 & 2.4 & 1.3 & 1.2 & 2.2 & 28.7 & & $\begin{array}{l}\text { ruderal places, fallow land, } \\
\text { balks }\end{array}$ \\
\hline Aster type & 0.4 & 1.2 & 1.0 & 2.1 & 0.2 & & 2.5 & Leontodon autumnalis & $\begin{array}{l}\text { meadows, pastures, path } \\
\text { margins }\end{array}$ \\
\hline Brassicaceae & 0.5 & 1.8 & 1.0 & 0.7 & 1.2 & 0.2 & 0.2 & $\begin{array}{l}\text { Camelina microcarpa, } \\
\text { Camelina sativa, Neslia } \\
\text { paniculata, Capsella } \\
\text { bursa-pastoris, Sinapis } \\
\text { arvensis }\end{array}$ & $\begin{array}{l}\text { fields, fallow land, ruderal } \\
\text { places }\end{array}$ \\
\hline $\begin{array}{l}\text { Bupleurum falcatum } \\
\text { type }\end{array}$ & 0.1 & & & & & & & Bupleurum rotundifolium & fields, fallow land \\
\hline Calluna vulgaris & & 0.3 & 2.1 & 0.2 & & & & & pastures, dry forests, rocks \\
\hline Calystegia sepium & 0.1 & & & & & & & & bank vegetation \\
\hline Cannabis sativa & 0.5 & 0.4 & 0.5 & 0.2 & & 0.2 & & Cannabis sativa & fields \\
\hline Centaurea cyanus & & 0.1 & & & & 0.1 & & & fields \\
\hline $\begin{array}{l}\text { Centaurea jaceal } \\
\text { C. stoebe }\end{array}$ & 0.8 & 0.7 & 1.7 & 2.1 & & 0.1 & 3.3 & Centaurea jacea & meadows, dry grasslands \\
\hline Centaurea scabiosa & 0.5 & 0.3 & & & & & & & dry grasslands \\
\hline Cerastium & & 0.1 & 0.5 & & & 0.2 & & & meadows, grasslands \\
\hline Cerealia & 2.8 & 19.7 & 29.0 & 53.2 & 4.0 & 13.8 & 3.7 & $\begin{array}{l}\text { Triticum, Avena sativa, } \\
\text { Panicum miliaceum, } \\
\text { Hordeum }\end{array}$ & fields \\
\hline Cirsium & 1.3 & 1.6 & 0.5 & 0.4 & & 0.3 & 4.1 & & $\begin{array}{l}\text { fields, fallow land, } \\
\text { ruderal places, moats, wet } \\
\text { meadows }\end{array}$ \\
\hline Consolida type & 0.2 & 0.3 & & & 0.2 & & & & fields \\
\hline Convolvulus arvensis & & & & 0.4 & 1.4 & 0.1 & & Convolvulus arvensis & fields, dry grasslands \\
\hline Cyperaceae & & & 0.7 & & & 0.7 & & $\begin{array}{l}\text { Carex hirta, Carex } \\
\text { spicata/muricata, } \\
\text { Eleocharis palustris } \\
\text { agg., Schoenoplectus } \\
\text { tabernaemontani }\end{array}$ & $\begin{array}{l}\text { path margins, ruderal } \\
\text { places, forest clearings, } \\
\text { shrubs, wetlands, banks }\end{array}$ \\
\hline Daucus carota & 0.1 & 1.4 & & & & & & & dry grasslands \\
\hline
\end{tabular}


Table 1. Results of pollen analysis confronted with the findings of plant macroremain analysis. Pollen types were defined according to Reille (1992), Beug (2004) and Punt (1980) (continued).

\begin{tabular}{|c|c|c|c|c|c|c|c|c|c|}
\hline Layer number & $\begin{array}{l}\infty \\
\sum_{0}^{\infty} \\
\infty \\
\bar{Z}\end{array}$ & 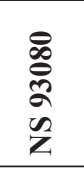 & 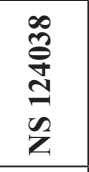 & 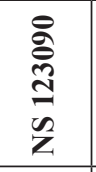 & 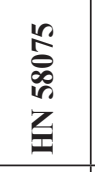 & 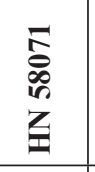 & 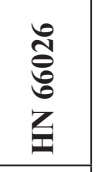 & & \\
\hline Archaeological dating & 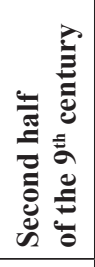 & 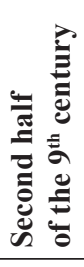 & 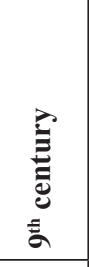 & 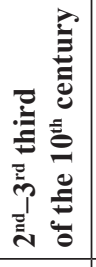 & 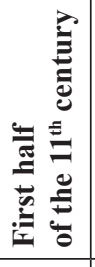 & 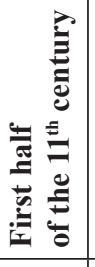 & 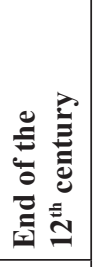 & & \\
\hline $\begin{array}{c}\text { Archaeological } \\
\text { context }\end{array}$ & 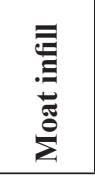 & 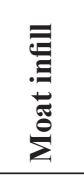 & 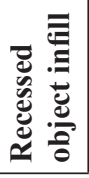 & 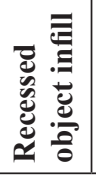 & 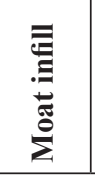 & 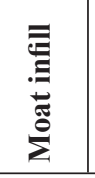 & 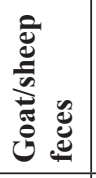 & & \\
\hline Pollen type & \multicolumn{7}{|c|}{$\%$ ratio in pollen spectrum } & Macroremain analysis & Prevailing type of biotope \\
\hline Dianthus type & & & 0.2 & & & & & & meadows, dry grasslands \\
\hline Dipsacus type & & & 0.2 & & & & & & $\begin{array}{l}\text { ruderal places, forest } \\
\text { clearings }\end{array}$ \\
\hline Euphorbia & & & & & & & 0.2 & & $\begin{array}{l}\text { dry grasslands, ruderal } \\
\text { places }\end{array}$ \\
\hline $\begin{array}{l}\text { Fallopia convolvulus/ } \\
\text { F.dumetorum }\end{array}$ & & & 0.5 & & & & & Fallopia convolvulus & $\begin{array}{l}\text { fields, fallow land, ruderal } \\
\text { places }\end{array}$ \\
\hline Fenestratae & 5.5 & 11.7 & 2.6 & 0.2 & 0.8 & 1.8 & & Lapsana communis & $\begin{array}{l}\text { fields, fallow land, ruderal } \\
\text { places }\end{array}$ \\
\hline $\begin{array}{l}\text { Filipendula ulmarial } \\
\text { F. vulgaris }\end{array}$ & 0.5 & 0.1 & 3.6 & 0.7 & 0.2 & & 0.4 & & $\begin{array}{l}\text { wet meadows and moats/ } \\
\text { dry grasslands }\end{array}$ \\
\hline Galeopsis-Ballota type & 0.4 & 0.7 & 0.7 & 0.2 & & & 0.2 & $\begin{array}{l}\text { Ajuga genevensis, } \\
\text { Galeopsis angustifolia, } \\
\text { Galeopsis tetrahit, } \\
\text { Lamium purpureum, } \\
\text { Stachys arvensis/annua }\end{array}$ & $\begin{array}{l}\text { meadows, fallow land, } \\
\text { shrubs, balks, fields, ruderal } \\
\text { places }\end{array}$ \\
\hline Genista type & 0.1 & & & & & & & & $\begin{array}{l}\text { dry grasslands, dry forest } \\
\text { margins }\end{array}$ \\
\hline Gramineae & 21.6 & 11.7 & 34.4 & 19.7 & 35.8 & 15.6 & 11.6 & $\begin{array}{l}\text { Agropyron repens, Agrostis } \\
\text { sp., Bromus arvensis, } \\
\text { Bromus secalinus, Bromus } \\
\text { sterilis, Echinochloa } \\
\text { cruss-gali, Setaria pumila, } \\
\text { Setaria viridis/verticilata } \\
\end{array}$ & $\begin{array}{l}\text { fields, fallow land, ruderal } \\
\text { places, dry grasslands }\end{array}$ \\
\hline Helianthemum & & 0.1 & & & & & & & dry grasslands \\
\hline Humulus lupulus & & & & 0.4 & & & & & shrubs, bank vegetation \\
\hline $\begin{array}{l}\text { Hypericum perforatum } \\
\text { type }\end{array}$ & 1.5 & 0.9 & & & & 0.7 & 0.2 & Hypericum perforatum & $\begin{array}{l}\text { dry grasslands, forest } \\
\text { clearings }\end{array}$ \\
\hline Chelidonium & 0.1 & & & & & & & & ruderal places \\
\hline Chenopodiaceae & 7.8 & 5.9 & 1.9 & 4.5 & 1.2 & 0.9 & 1.4 & $\begin{array}{l}\text { Atriplex patula, } \\
\text { Chenopodium album, } \\
\text { Chenopodium hybridum, } \\
\text { Chenopodium murale, } \\
\text { Chenopodium } \\
\text { polyspermum }\end{array}$ & $\begin{array}{l}\text { ruderal places, waste } \\
\text { deposits }\end{array}$ \\
\hline $\begin{array}{l}\text { Linum usitatissimum } \\
\text { type }\end{array}$ & & & & & & 0.7 & & Linum usitatissimum & fields \\
\hline Lotus & & 0.1 & & & & & & & meadows, dry grasslands \\
\hline
\end{tabular}


Table 1. Results of pollen analysis confronted with the findings of plant macroremain analysis. Pollen types were defined according to Reille (1992), Beug (2004) and Punt (1980) (continued).

\begin{tabular}{|c|c|c|c|c|c|c|c|c|c|}
\hline Layer number & 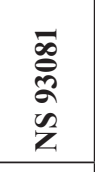 & 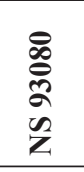 & 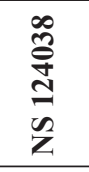 & 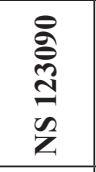 & 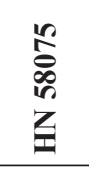 & $\begin{array}{l}5 \\
5 \\
\infty \\
10 \\
z \\
1\end{array}$ & 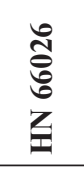 & & \\
\hline Archaeological dating & 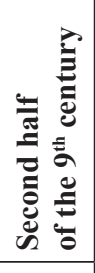 & 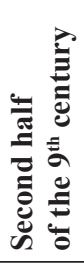 & 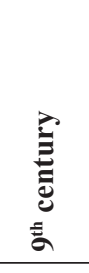 & 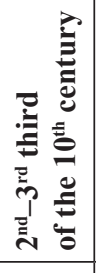 & 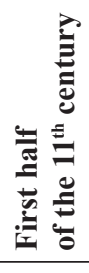 & 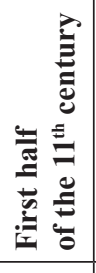 & 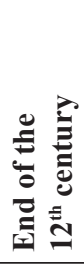 & & \\
\hline $\begin{array}{l}\text { Archaeological } \\
\text { context }\end{array}$ & 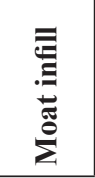 & 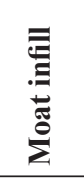 & 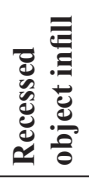 & 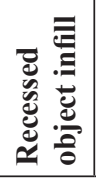 & 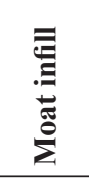 & 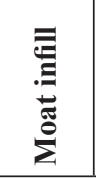 & 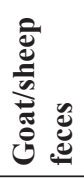 & & \\
\hline Pollen type & \multicolumn{7}{|c|}{$\%$ ratio in pollen spectrum } & Macroremain analysis & Prevailing type of biotope \\
\hline Matricaria type & 1.4 & 1.7 & 1.2 & & & & 9.9 & Anthemis arvensis & $\begin{array}{l}\text { fields, fallow land, ruderal } \\
\text { places }\end{array}$ \\
\hline Melampyrum & 0.1 & & & 0.9 & & & 0.2 & & fields, dry grasslands, forests \\
\hline Mentha type & & 0.1 & 0.2 & & & 0.1 & & & $\begin{array}{l}\text { fallow land, dry grasslands, } \\
\text { wetlands, moats }\end{array}$ \\
\hline Odontites type & 0.2 & & & & & & & & $\begin{array}{l}\text { dry grasslands, path } \\
\text { margins, pastures }\end{array}$ \\
\hline Onobrychis & 0.1 & & & & & & & & dry grasslands, balks \\
\hline Papaver rhoeas type & & 0.4 & 0.2 & 3.1 & 1.3 & 1.3 & & $\begin{array}{l}\text { Glaucium corniculatum, } \\
\text { Papaver somniferum } \\
\end{array}$ & fields, dry grasslands \\
\hline $\begin{array}{l}\text { Persicaria maculosa } \\
\text { type }\end{array}$ & & & & & & 0.7 & & Persicaria lapathifolia & ruderal places \\
\hline Plantago lanceolata & 18.3 & 6.5 & 1.2 & 1.1 & 1.9 & 0.9 & 1.4 & Plantago lanceolata & $\begin{array}{l}\text { pastures, meadows, } \\
\text { ruderalized grasslands }\end{array}$ \\
\hline Plantago major & & 0.3 & & & 0.2 & & 0.2 & & $\begin{array}{l}\text { pastures, ruderal places, } \\
\text { path margins }\end{array}$ \\
\hline Plantago media & 0.5 & 0.3 & 0.7 & & 0.3 & 0.5 & & & meadows, pastures \\
\hline Polygonum aviculare & 0.1 & 0.1 & 0.2 & & & & & Polygonum aviculare & down trodden places \\
\hline Potentilla/Fragaria & & 0.1 & 0.2 & & & & 0.6 & $\begin{array}{l}\text { Fragaria vesca, } \\
\text { Potentilla supina }\end{array}$ & $\begin{array}{l}\text { forst clearings, shrubs, } \\
\text { ruderal places, banks }\end{array}$ \\
\hline $\begin{array}{l}\text { Ranunculus sceleratus } \\
\text { type }\end{array}$ & & & & 0.7 & 0.2 & 0.1 & 0.2 & & ruderal places \\
\hline Ranunculus acris type & & 0.1 & 0.2 & 0.2 & 0.3 & 0.7 & 0.6 & Ranunculus acris & wet meadows \\
\hline Rubiaceae & 0.5 & 0.3 & 1.0 & 0.4 & 0.8 & 0.5 & & $\begin{array}{l}\text { Galium aparine, Galium } \\
\text { spurium }\end{array}$ & $\begin{array}{l}\text { shrubs, ruderal places, } \\
\text { fallow land, fields }\end{array}$ \\
\hline Rubus & 0.1 & & & & & & & $\begin{array}{l}\text { Rubus caesius, Rubus } \\
\text { fruticosus agg., Rubus, } \\
\text { idaeus }\end{array}$ & $\begin{array}{l}\text { shrubs, forest margins, } \\
\text { forest clearings }\end{array}$ \\
\hline Rumex acetosa type & & & 0.2 & & & & & Rumex crispus & $\begin{array}{l}\text { beds, ruderal places, fallow } \\
\text { land }\end{array}$ \\
\hline Sagina nodosa type & & & & & 0.2 & & & Sagina procumbens & wet down trodden places \\
\hline $\begin{array}{l}\text { Scabiosa columbaria } \\
\text { type }\end{array}$ & & & & 0.4 & 0.8 & 0.7 & 0.2 & & dry grasslands \\
\hline Scleranthus annuиs & 0.5 & & & & 0.2 & & & Scleranthus annuus & fields \\
\hline Scrophulariaceae & 0.2 & 0.1 & & & & & 3.3 & & $\begin{array}{l}\text { dry grasslands, ruderal } \\
\text { places, banks }\end{array}$ \\
\hline Secale cereale & 0.2 & 0.5 & & & 13.8 & 0.3 & 1.4 & Secale cereale & fields \\
\hline Silene type & 0.5 & 0.4 & & & 0.3 & 0.3 & & & dry grasslands \\
\hline
\end{tabular}


Table 1. Results of pollen analysis confronted with the findings of plant macroremain analysis. Pollen types were defined according to Reille (1992), Beug (2004) and Punt (1980) (continued).

\begin{tabular}{|c|c|c|c|c|c|c|c|c|c|}
\hline Layer number & $\begin{array}{l}\bar{\infty} \\
\stackrel{0}{\sigma} \\
\infty \\
z\end{array}$ & 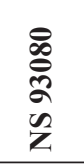 & 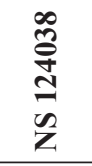 & 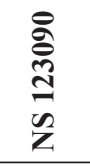 & 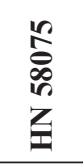 & 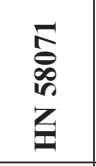 & 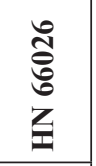 & & \\
\hline Archaeological dating & 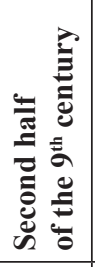 & 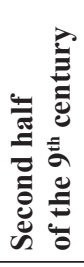 & 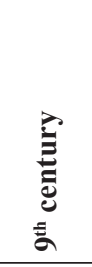 & 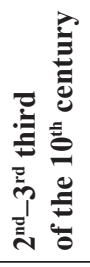 & 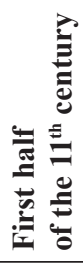 & 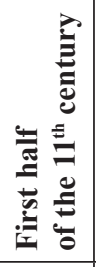 & 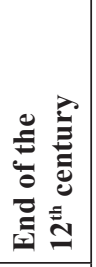 & & \\
\hline $\begin{array}{c}\text { Archaeological } \\
\text { context }\end{array}$ & 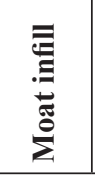 & 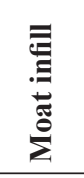 & 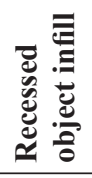 & 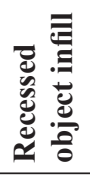 & 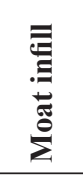 & 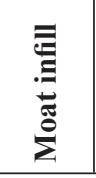 & 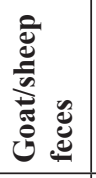 & & \\
\hline Pollen type & \multicolumn{7}{|c|}{$\%$ ratio in pollen spectrum } & Macroremain analysis & Prevailing type of biotope \\
\hline Thalictrum & & & 0.2 & & & & 1.0 & & wet meadows \\
\hline Trifolium pratense type & & 0.5 & & & & & 0.8 & Trifolium pratense & meadows, pastures \\
\hline Trifolium repens type & 0.1 & 0.4 & & & & & & Trifolium repens & $\begin{array}{l}\text { pastures, grasslands, path } \\
\text { margins }\end{array}$ \\
\hline Urtica & 1.2 & 0.4 & & & 0.6 & 0.2 & 0.2 & & $\begin{array}{l}\text { banks, ruderal places, waste } \\
\text { deposits }\end{array}$ \\
\hline Valeriana officinalis & 0.4 & & 1.0 & & & & & & $\begin{array}{l}\text { wet meadows and moats, } \\
\text { banks }\end{array}$ \\
\hline Veronica type & 0.1 & & & & & 0.7 & & & $\begin{array}{l}\text { dry grasslands, ruderal } \\
\text { places, fallow land, path } \\
\text { margins }\end{array}$ \\
\hline Vicia type & & & & & & & 0.2 & & \\
\hline Xanthium & & 0.1 & & & & & & & path margins, ruderal places \\
\hline monolete spore & 0.1 & 3.5 & 0.5 & & & 0.7 & & & forests, carrs \\
\hline Chaetomium & 0.1 & 0.9 & & & 0.6 & 56.6 & & & $\begin{array}{l}\text { celulose decomposing fungi } \\
\text { - wet eutrophic conditions }\end{array}$ \\
\hline Podospora & & & & & 0.2 & & 0.4 & & coprophilous fungi \\
\hline Sporomiella & 0.1 & & & & 0.6 & & 0.2 & & coprophilous fungi \\
\hline Thecaphora & 0.5 & 1.8 & 0.7 & 2.1 & 1.3 & & & & $\begin{array}{l}\text { parazitic fungi - often on } \\
\text { Fabaceae }\end{array}$ \\
\hline Trichuris & 0.2 & 0.5 & & & & & 0.4 & & mammal intestine parazite \\
\hline Sum $100 \%$ & 976 & 768 & 421 & 447 & 447 & 1483 & 484 & & \\
\hline
\end{tabular}

\section{Results and discussion}

On the basis of an examination of archaeobotanical material this study aims to improve knowledge of what we believe are the four key steps that will lead to a better understanding of the economic situation at Žatec and its agricultural background. Including:

- To define the range of cultivated plants.

- To specify the most economically important arable crops.

- To trace changes in ratios of these crops in time.

- To try to identify changes in agricultural technology and discuss the contemporaneous mainstream agricultural system.
A total of 101 samples were analyzed by systematic sampling of well-dated archaeological layers (especially the infill of objects that contained a sufficient amount of chronologically sensitive pottery material). A total of 15,042 plant macroremains were identified. This set of plant remains corresponds to approximately 140 well determined types of plants, out of which 42 (approximately 30\%) were cultivated.

\subsection{The range of cultivated plants, changes in time and associations with particular agricultural systems}

The examined set of plant macroremains contained approximately 20 domesticated plants, about 10 types of gathered wild plants and 12 possibly cultivated plants. 
A large number of archaeobotanical analyses have been conducted in Europe covering the period of the $9^{\text {th }}$ to $10^{\text {th }}$ centuries. Thus, our set of plant macroremains resulted in minimal new (e.g. newly discovered) findings of cultivated species. ${ }^{1}$ However, our archaeobotanic data are especially valuable in that they form a systematically sampled set that gives us more specific information about the contemporary importance of individual cultivated species.

The presence or absence of a particular species in the samples was used as an indicator of their importance. Figure 2 shows the number of samples in which a given type was present.

When evaluating changes in the range of cultivated species, we face the problem of different archaeological preservation of individual cultivated species or groups. Whereas cereal crops make up a majority of the carbonized plant remains, they are almost absent from mineralized remains. Similarly, macroremains of garden produce are very rare in carbonized assemblages, but may be found in large volumes of non-carbonized plant macroremains. This difference in depositional preservation may be explained by different kitchen processing of cereal crops. During such processing the grains may have been burnt and disposed of as waste. On the other hand, we may assume that garden produce was usually eaten without any heat processing. The resistance of the seeds and fruits of individual species (or group of species) with respect to decay, the content of mineral salts in macroremains, as well as the ability to carbonize during the burning process all influence the degree and quality of macroremain preservation.

When evaluating the level and changes in the economy of Žatec and its agricultural background, field crops (cereal crops and pulses) are especially important. This group of cultivated species was dominant in the preserved set of carbonized macroremains, again demonstrating the primary importance of carbonized macroremains for analyses of archaeological data (see above).

Changes in the proportions of cereal crops and weeds over time provided the first indicator of changes in the agricultural system of the Early Medieval period. One indicator of the transition from the older two-field rotation to a three-field system is in the change of importance of individual cereal crops. Whereas a dominance of naked wheat can be observed in the Early Medieval period, the growing importance of rye is observed from the High Medieval period onwards.

This general trend was noted in early and recent research by Beranová (1975) and Beranová, Lutovský (2008). She observed that cultivation of millet and wheat prevailed in the $8^{\text {th }}$ to $12^{\text {th }}$ centuries, and that a substantial change occurred at the beginning of the $13^{\text {th }}$ century with the introduction

${ }^{1}$ Massive finds of Camelina alyssum are surprising, since this species is the oldest evidence of a flax-accompanying weed in our territory. The discovery of the relatively rare xerophyte and thermophilic Stipa, and the relatively frequent identification of Vitis vinifera are good indications that vine was cultivated around Žatec from the $9^{\text {th }}$ to $10^{\text {th }}$ centuries: Žatec B-C. of more efficient instruments for ploughing and harvesting, large-scale colonization, and a massive transition from twofield to three- field rotation. Cereal production changed radically in favour of rye, while wheat and millet went into decline (Beranová 1975).

It is still not clear whether this transition also occurred in the more fertile lowlands or whether the increase in rye was the result of human migration into regions with less fertile soils. According to František Graus (1957) who examined written sources of the $13^{\text {th }}$ and $14^{\text {th }}$ centuries, rye had a dominant position and "wheat was cultivated in a lesser amount, and namely in the most fertile parts of Bohemia".

When studying currently available results of archaeobotanical analyses of cereal crops from the lowlands of medieval Bohemia and Moravia, we find that the transition into "rye" production may be clearly identified in the High Medieval period but that data during the $11^{\text {th }}$ and $12^{\text {th }}$ centuries are almost completely missing (Figure 3 ).

Considering the agricultural changes in Žatec we hypothesise that the increased proportion of rye in cereal crop production reflects a transition to a system which is very similar to the high medieval three-field rotation. Archaeobotanical findings from Žatec indicate a change in the agricultural system, they are not, however, necessarily proof of a concrete system of field cultivation. We do not yet assume the existence of a truly regulated and unified three-field rotation, which required the transformation of country settlement connected with High Medieval period colonization.

Figures 4 and 5 show the set of carbonized macroremains of cultivated plants divided into four periods on the basis of archaeological dating. Samples with an extremely wide interval and/or inaccurate dating were excluded. The data may be separated into two contrasting intervals based on changes in the range of cultivated plants: the $9^{\text {th }}$ and $10^{\text {th }}$ centuries, and the $11^{\text {th }}$ and $12^{\text {th }}$ centuries, respectively. The first interval ( $9^{\text {th }}$ and $10^{\text {th }}$ centuries) is characterised by the unambiguous dominance of naked Triticum aestivum, in combination with Triticum compactum, whose grains can not be explicitly differentiated from the Triticum aestivum. The proportion of other cereal crops (barley, oats, bearded wheats) during these periods does not exceed $5 \%$, with the exception of the oldest phase in which archaic bearded wheats (Triticum dicoccon, $T$. cf. spelta) account for more than $16 \%$ of the total. This high proportion of bearded wheats is unusual for the Early Medieval period, and may be explained by either the relatively low number of data from this chronological time period (151 items), or by the possibility of contamination by older prehistoric material. Early Bronze Age settlement is clearly manifested at Žatec. Early Medieval period objects are often recessed into prehistoric layers, and the Medieval layers with pottery from the Žatec B and B-C time periods ( $9^{\text {th }}$ century) are situated on top of prehistoric layers. Early Medieval pottery is often found together with pottery from the Late Bronze Age. Amongst pulses, there is a higher proportion of cultivated lentil (Lens culinaris), especially in the oldest period of the $9^{\text {th }}$ century. In the same layers there are also higher amounts of Cannabis sativa macroremains. 


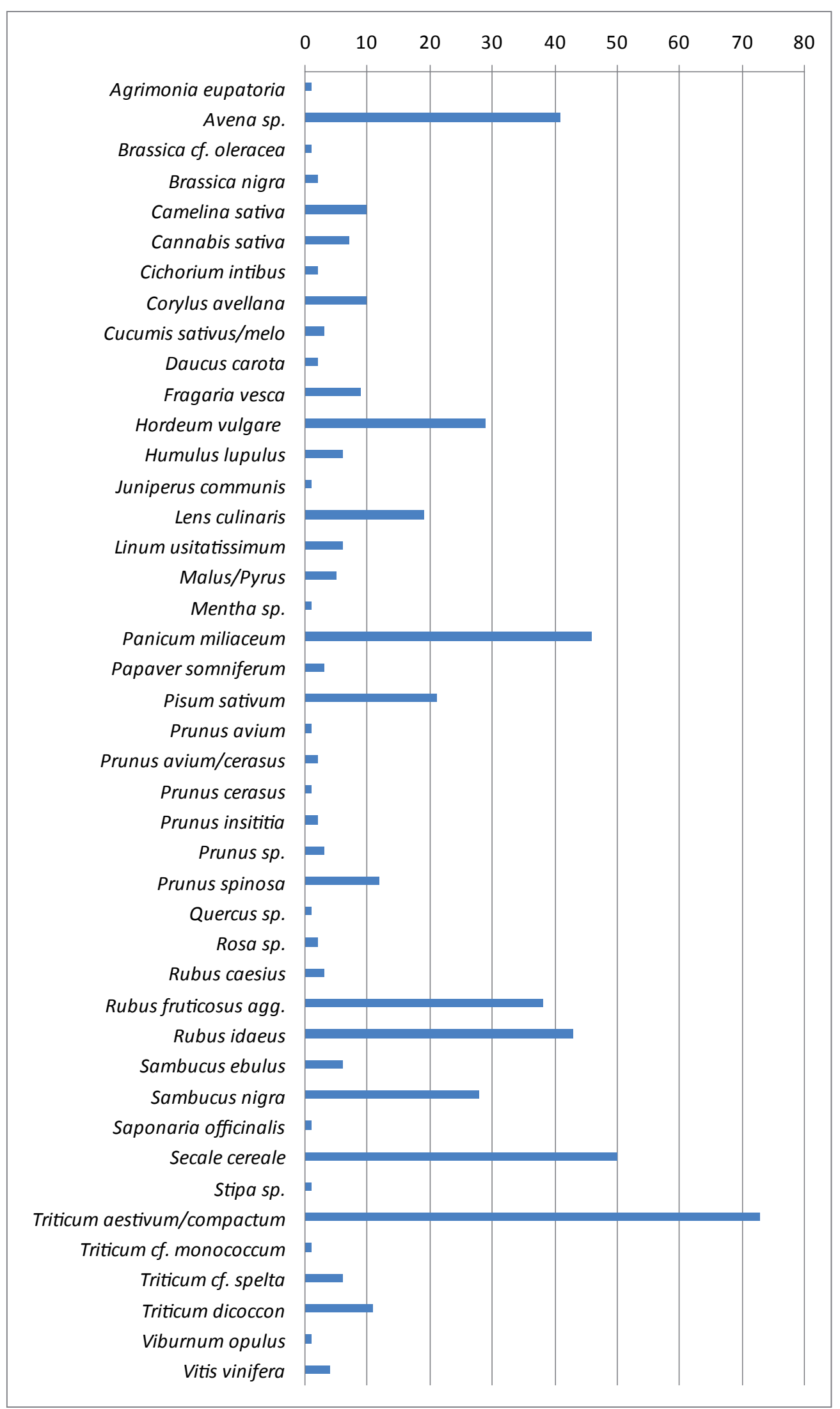

Figure 2. Early Medieval period Žatec - the importance of particular cultivated and gathered species, expressed as the number of samples containing each particular species ( $\mathrm{n}=101$ samples). 


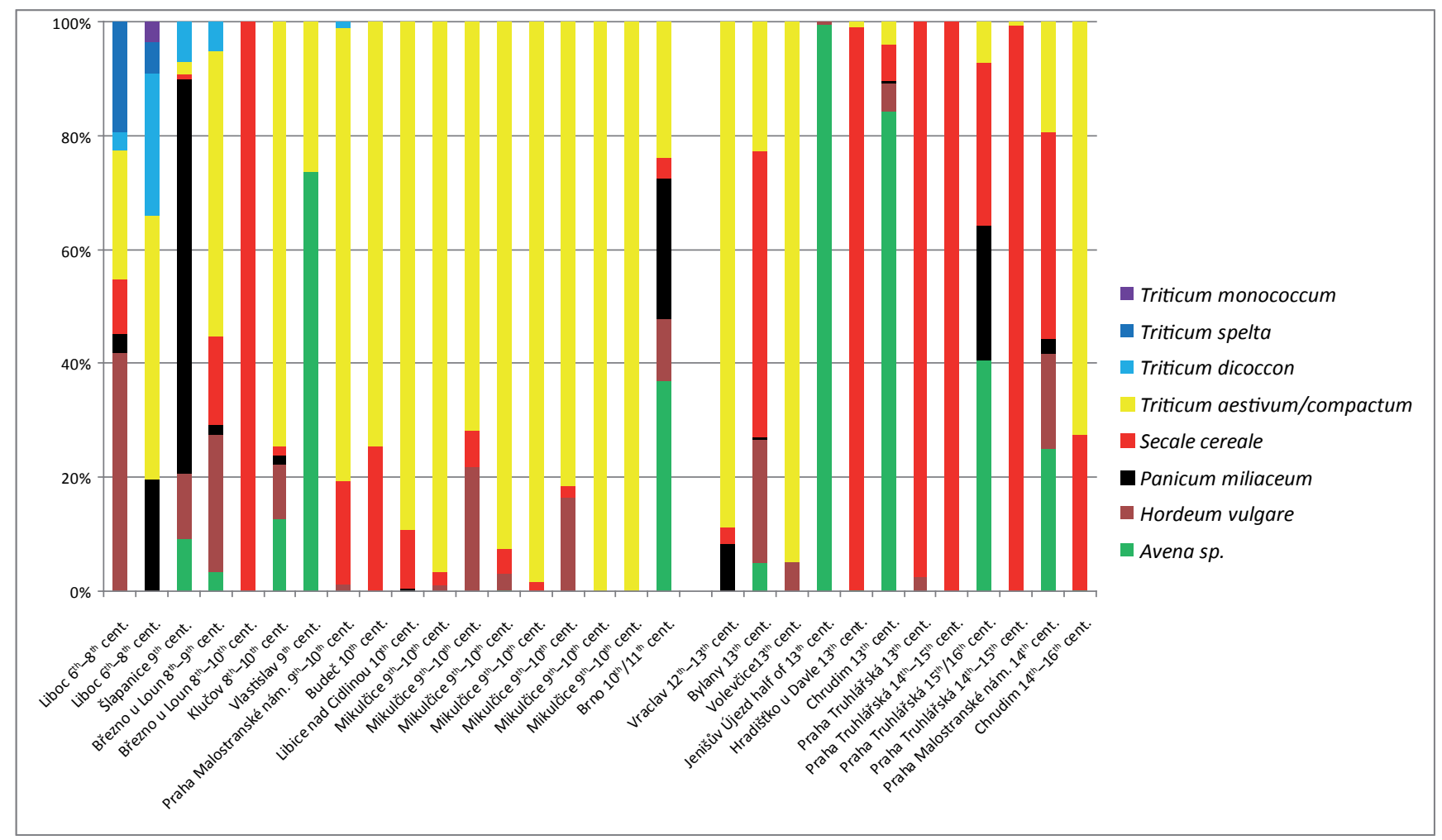

Figure 3. Medieval cereal crops in the Czech Republic; all standard quantified results (numerically defined) from archaeobotanical analyses with grain numbers exceeding 30 are given.

A considerable change in the set of cereal crops is found in the $11^{\text {th }}$ and $12^{\text {th }}$ centuries. Naked wheats (likely common wheat) still prevail but are accompanied by a high proportion of cultivated rye (Secale cereale), and a higher proportion of other relatively rare cereal crops, such as common barley (Hordeum vulgare) and oats (Avena sp.). A certain change is also evident among pulses, with the field pea (Pisum sativum) becoming more numerous.

We consider changes in the range of cereal crops to be crucial. We monitored increase of rye also in the data with exclusion of all mass findings of cereal crops exceeding 1,000 pieces of grains that might have strongly affected

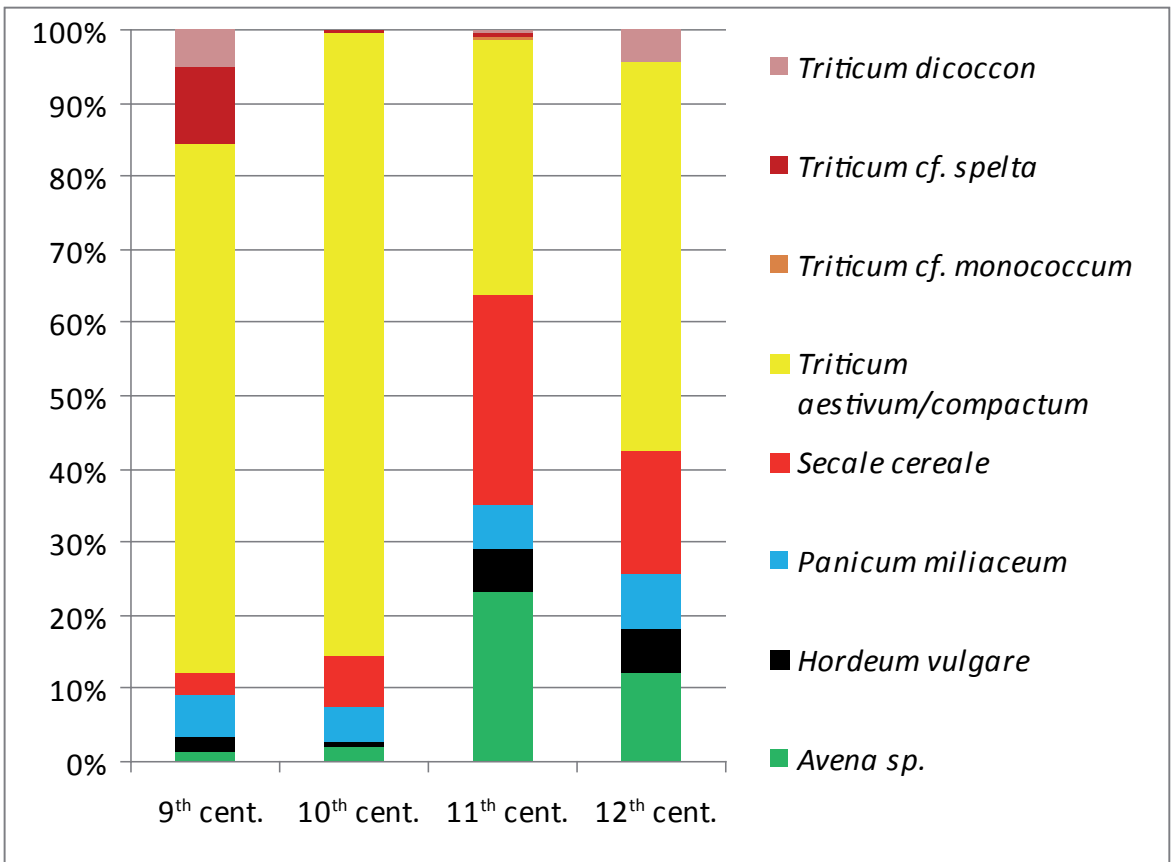

Figure 4. Early Medieval period Žatec proportionsofmaincerealcropmacroremains: excluding samples with over 1,000 items, and oats where wild and cultivated species can not be clearly differentiated (only carbonized macroremains, $n=2,544$ ). 
Figure 5. Early Medieval period Žatec evidence of wheat and rye macroremains in individual periods (only carbonized macroremains, $\mathrm{n}=101$ ).
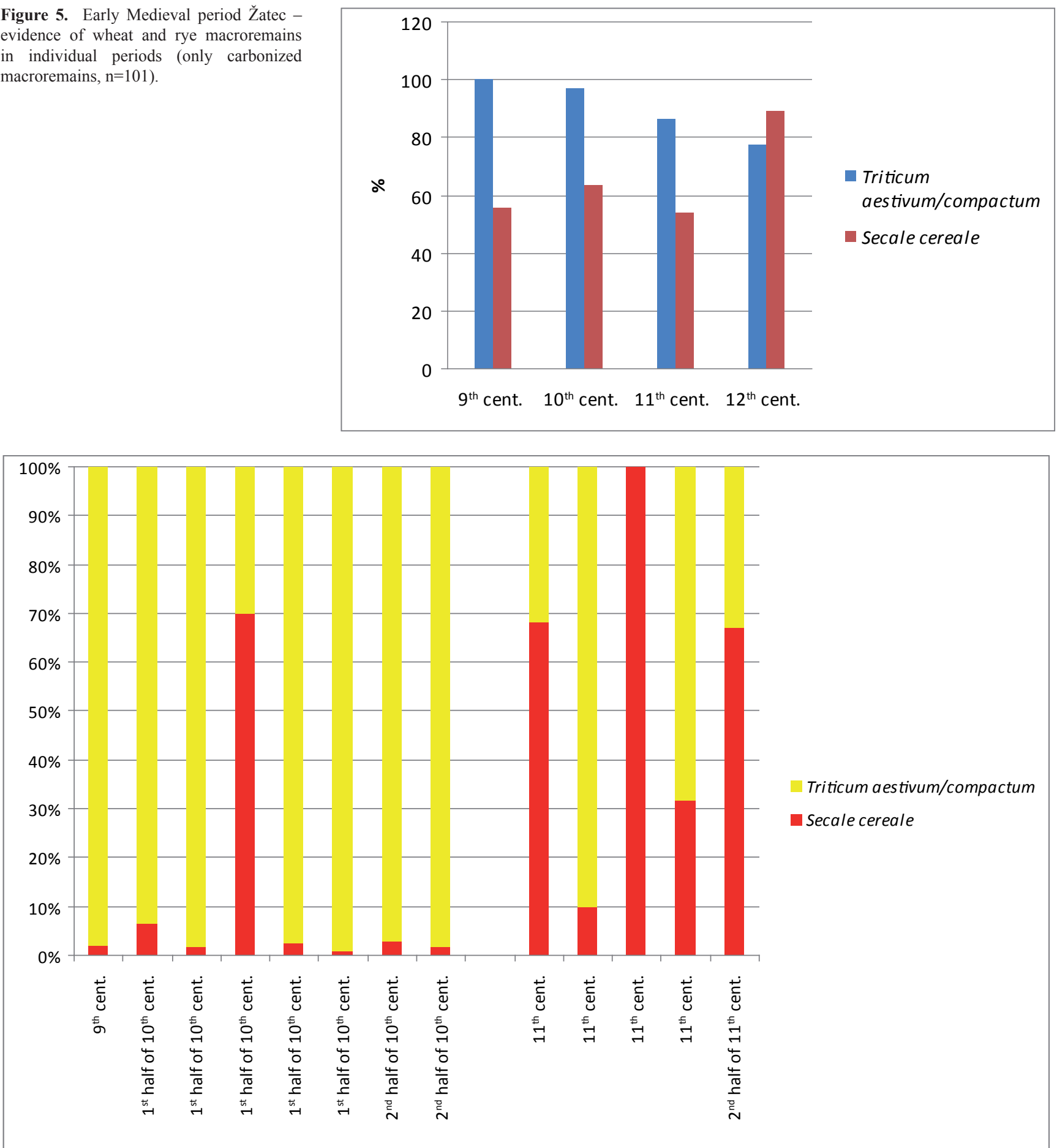

Figure 6. Žatec - evidence of wheat and rye macroremains in samples where the number of macroremains of these species exceeds 40 items. $9^{\text {th }}$ century. -

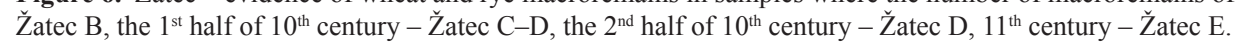

proportions of cereal crops. The increased importance of rye was observed in an absolute number of grains in samples, in number of samples where rye was present (time period 4) and even among mass findings of cereal crops.

This archaeobotanical dataset, especially the carbonized items, contains a considerable number of field weeds mixed in with cultivated species. The range and type of field weeds are very sensitive indicators of conditions during the cultivation and processing of field produce.
It may be assumed from recent studies that rye stands are accompanied by typical winter weeds (especially species such as Agrostemma githago, Bromus secalinus, Bromus arvensis, etc.). Winter wheat stands are also accompanied by these winter weeds, but to a lesser extent and with a higher numbers of summer weeds.

Similarly as for other field weed macroremain counts from the Early Medieval period, the set from Žatec contains a majority of summer weeds versus winter weeds, and 

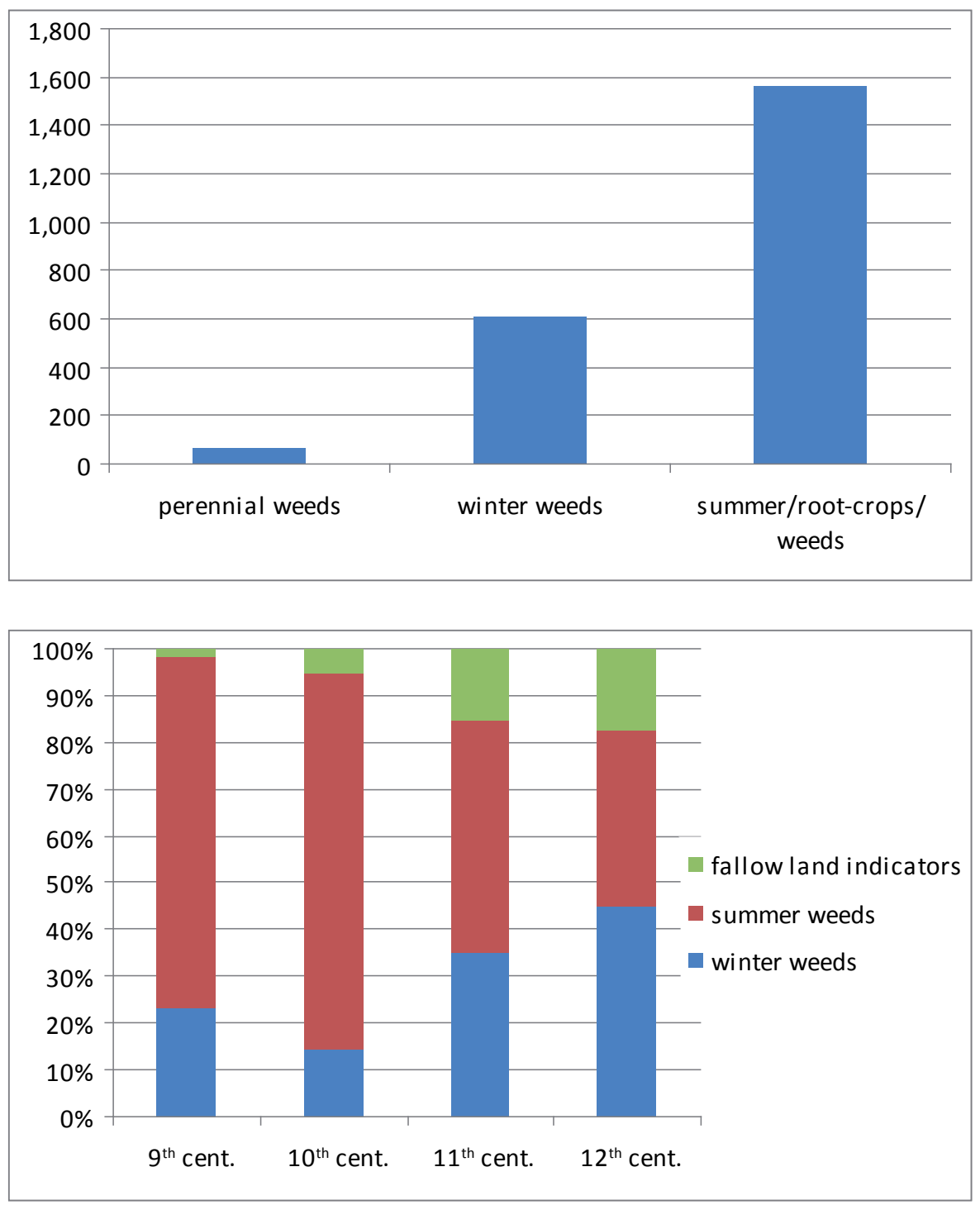

Figure 7. Early Medieval period Žatec - rates of three groups of weeds (only carbonized macroremains, $\mathrm{n}=2,401$ ).

Figure 8. Early Medieval Žatec proportions of different weed groups (only carbonized macroremains, $n=2,267$ ). perennial weeds were relatively rare. This is probably caused by the fact that summer weeds also occur quite frequently in winter produce, while just some species such as Adonis aestivalis, Agrostemma githago, Bromus secalinus or Vicia hirsuta are reliable indicators of winter produce.

As with the field produce, there are substantial changes in weed taxa between periods. The first and second period ( $9^{\text {th }}$ and $10^{\text {th }}$ centuries) show a high rate of summer weeds (approx. 75\%), while winter weeds form only a lesser part of these layers $\left(23 \% 9^{\text {th }}\right.$ and $14 \%$ in $10^{\text {th }}$ century, respectively). On the other hand there was a considerable increase in winter weeds during the $11^{\text {th }}$ and $12^{\text {th }}$ centuries $\left(35 \%\right.$ in the $11^{\text {th }}$ and $44 \%$ in the $12^{\text {th }}$ ), while the amount of summer weeds falls to $49 \%$ in the $11^{\text {th }}$ century, and to $37 \%$ in the $12^{\text {th }}$ century. The increasing proportion of species that grow on fallow land (plant species of fallow and ruderalised lands) is also striking, especially in the $11^{\text {th }}$ and $12^{\text {th }}$ centuries (from less than $2 \%$ in the $9^{\text {th }}$ century they increase to almost $18 \%$ during the $12^{\text {th }}$ century; Figure 8 ).

Changes in weed communities correlate with changes in the range of cereal crops (see above). During the $9^{\text {th }}$ and $10^{\text {th }}$ centuries, some features of archaic agriculture are still present, focused on the cultivation of naked wheat and connected with high rates of summer weeds compared to winter weed communities and fallow land taxa. Throughout the subsequent $11^{\text {th }}$ and $12^{\text {th }}$ centuries, the proportions of winter weeds, summer weeds and fallow land taxa are much more similar (Figure 7).

\subsection{Pollen spectra of cultural sediments}

Only six layers were found suitable for pollen analysis during the excavation. The continuously drying aerated sediments that predominate in the sub-soil of Žatec are not suitable for preserving pollen grains. The analyzed samples extend to the period between the $9^{\text {th }}$ and the second half of the $10^{\text {th }}$ centuries (Table 1).

Analysed layers, were part of the moat and other objects infill, and were probably the result of accumulations of various waste. This theory is supported by the spectrum of identified species, with a considerable prevalence of synanthropic taxa, weeds and crops (Figure 9). The relatively rare occurrence of ovum shells of the intestinal parasite Trichuris may indicate 


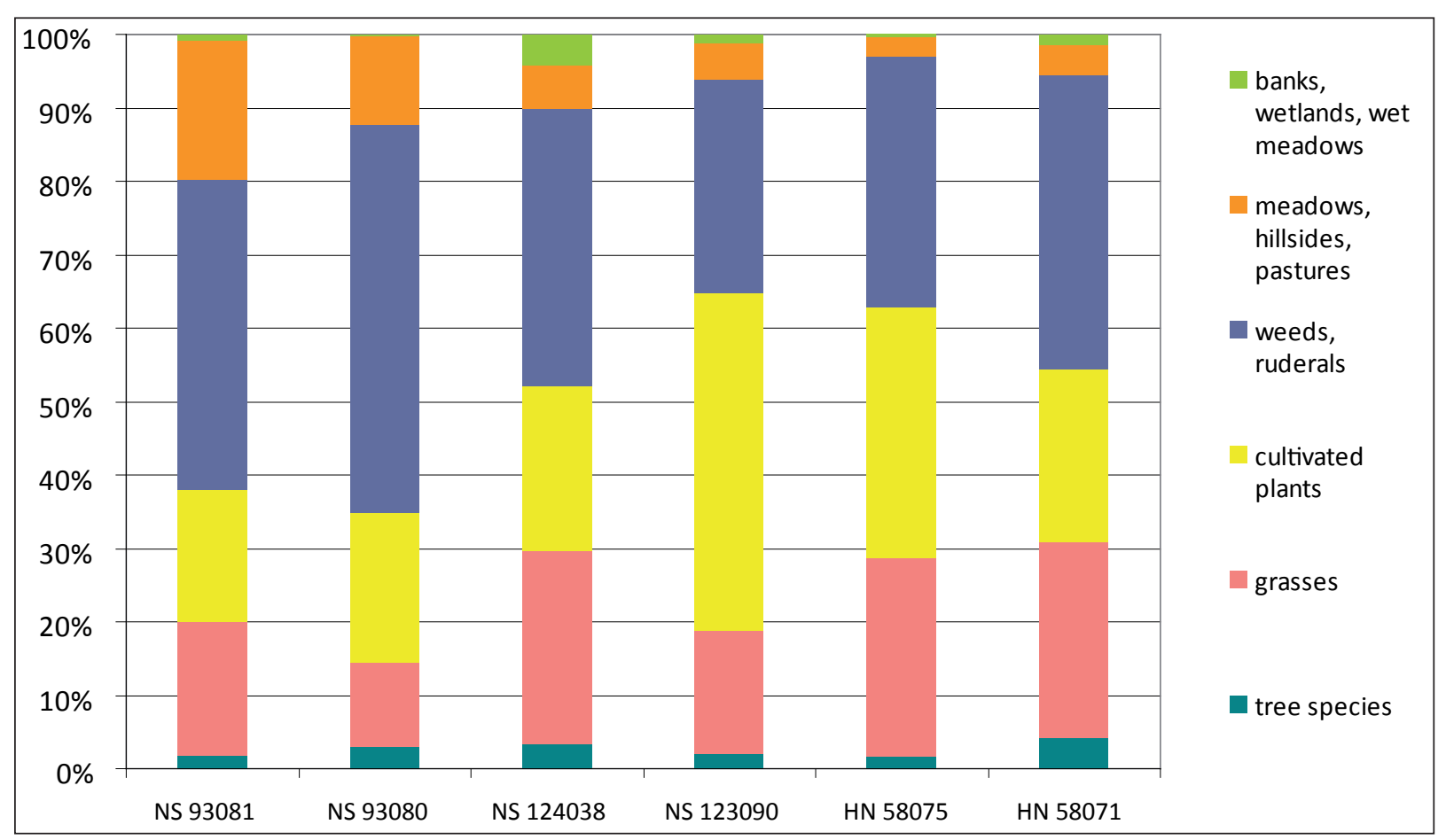

Figure 9. Proportions of particular ecological types of vegetation in the pollen spectra. $9^{\text {th }}$ century $-1^{\text {st }}$ half of $10^{\text {th }}$ century (time period of pottery Žatec C, C-D): NS 93081, NS 93080, NS 124038, 123090; $11^{\text {th }}$ century (time period of pottery Žatec E): HN 58075, HN 58071. NS - Náměstí Svobody square, HN - Hoštálkovo square.

that faecal waste was not deposited at the bottom of the moat, or that the inhabitants of the town were not seriously infected by this parasite.

The proportion of tree species ranges around $5 \%$ in the majority of samples. The pollen of tree species is deposited in cultural layers mostly naturally, i.e. transported by air. We may therefore assume that the identified types of trees came from fragmented forest vegetation, including beech and fir(Málek 1983). However, the forest vegetation in town layers from the Early Medieval period usually constitutes a substantial part of the pollen spectra. A good example can be seen in early medieval urban layers from Prague, where tree species generally constitute at least $10 \%$ and often more. On the other hand, in those instances the analysed layers were not waste sediments (Kozáková, Pokorný 2007; Kozáková, Boháčová 2008; Jankovská 1997, 1998). In layers from Žatec there are two probable factors that may have caused the low percentage of tree pollen - the prevailing human factor in the taphonomy of the sediments and extensive deforestation of the surrounding landscape.

It is notable that the pollen analysis, in comparison with the plant macroremains, shows more meadow and pasture taxa (Table 1). These may enter sediments through livestock excrement; indirect evidence for this theory is supported by spores of coprophilous fungi, (those that grow on excrement), Sporomiella and Podospora, that were identified in the analysed layers. Pollen of Anthericum and Onobrychis arenaria were also identified. These species are now relatively rare and are typical of sunny dry hillsides with stones - biotopes that are not influenced by any intensive human activity. The same conditions are suitable for other identified taxa such as Helianthemum, Dianthus, Centaurea scabiosa and Scabiosa. These species might have also grown on pastured areas, embankments or lawns within the city. More common taxa, such as Centaurea jacea/C. stoebe, Cerastium, Odontites or Lotus, possibly grew on places that were more affected by foot traffic, pasture, etc.

\subsection{Pollen spectrum from goat or sheep excrement}

Table 1 shows also the results of pollen analysis from excrement found in layer 66026 that constitutes the filling of a recessed object in sector 66 in Hoštálkovo square. Plant taxa of intensively ruderalized sites such as manor-houses are prevalent. The results reflect a short period of time during which an animal or animals were kept in some enclosed area within the town. It was most probably a goat, since the most frequent pollen taxa found in the diet, such as Artemisia, are not generally eaten by sheep. Animals most probably grazed ruderal vegetation growing on the site. Pollen grains of crops and trees (namely hazel and beech) indicate that animals were also given straw and branches with leaves (branch and twig foddering). This type of fodder supplementation is known to have occured since the Neolithic period (Rasmussen 1993; Akeret et al. 1999). In the case of Žatec the amount of arboreal pollen is relatively low since the landscape in the vicinity was extensively deforested, and branches with leaves were probably scarce commodities.

\subsection{Charcoal analysis}

Charcoal and wood remains are the final group of plant remains which was studied within this project. Analysis of non-carbonized wood provides information on the spectrum 


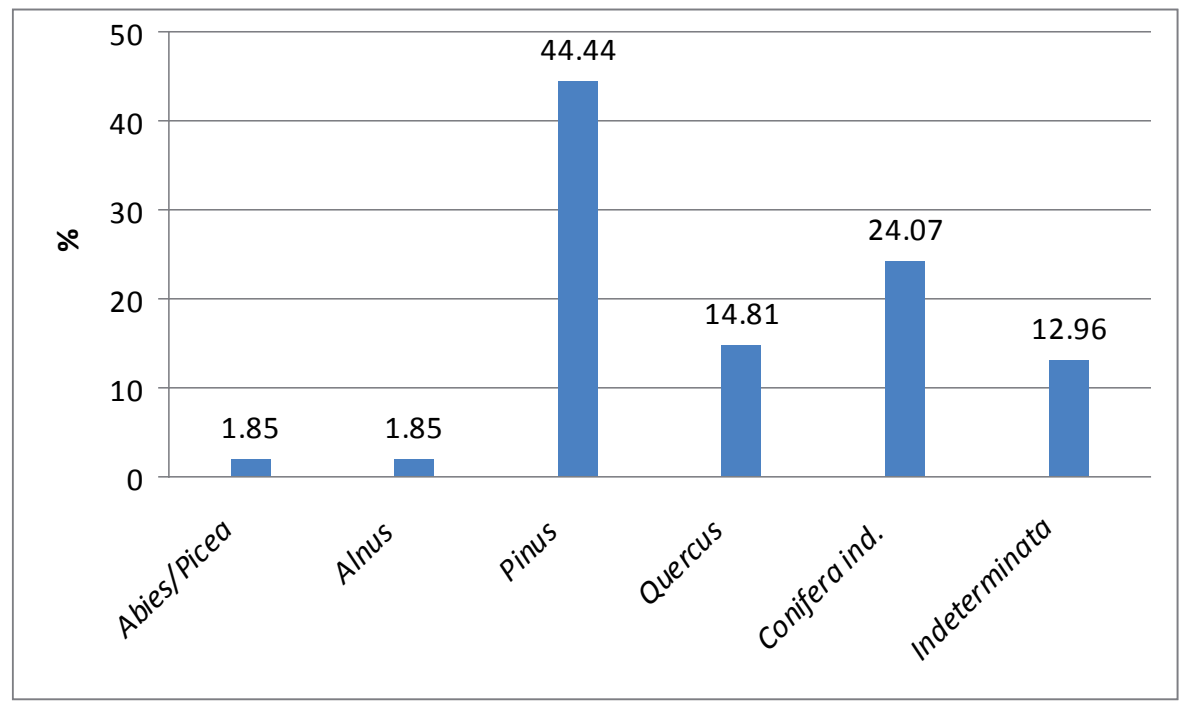

Figure 10. Early Medieval period Žatec results of analysis of noncarbonized wood $\mathrm{n}=54$.

of wood types used for construction purposes. Conifers (pine, fir/spruce and undefined coniferous species) made up a total of $70 \%$, followed by oak (up to $15 \%$ ) and marginal proportions of other wood (e.g. alder). As with pollen, wood was found in a small number of samples, since they are only preserved under ideal fossilisation conditions (consistently moist and anaerobic). Thus, there were only 7 samples of non-carbonized wood, taken from infill of recessed objects on Hošt’álkovo and Žižkovo squares.

Charcoal analysis mainly reflects the main types of firewood, and usually corresponds with tree species in local forests. The fact that most data is not influenced by fossilisation conditions is a great advantage.

Analysed charcoals from Žatec show the absolute dominance of oak and pine; other trees occurred infrequently $(<5 \%)$. These results indicate advanced deforestation of the fertile soils in the vicinity of Žatec, since oak forests with pines where such wood could be obtained, lie on acid soils and are not considered to be the potential dominant forest community in the area. According to soil and geological conditions, oak-hornbeam forests along with other more eutrophic phytocoenoses should have dominated the vegetation in the landscape around Žatec. It seems that wood for the early phase at Žatec was acquired from ecologically less-important, non- agrarian soils such as acid sandy subsoils, whereas soils of higher quality were mainly deforested and used for agricultural production.

In order to evaluate changes in the economy of the early medieval settlement, the species identified were divided into two groups according to their forest community relationships. The first group included all tree taxa which may be considered part of the local forest community (acid oak forests, oak-hornbeam forests, alluvial forests); the second group included the tree taxa that mainly occur at greater distances from Žatec - in higher altitudes (fir-beech forests, beech forests and spruce forests).

There was a decline of "local" trees (from almost 100\% in the oldest period down to approximately $73 \%$ in the youngest, Figure 12). Consequently, higher altitude tree species showed a gradual increase in the proportion of

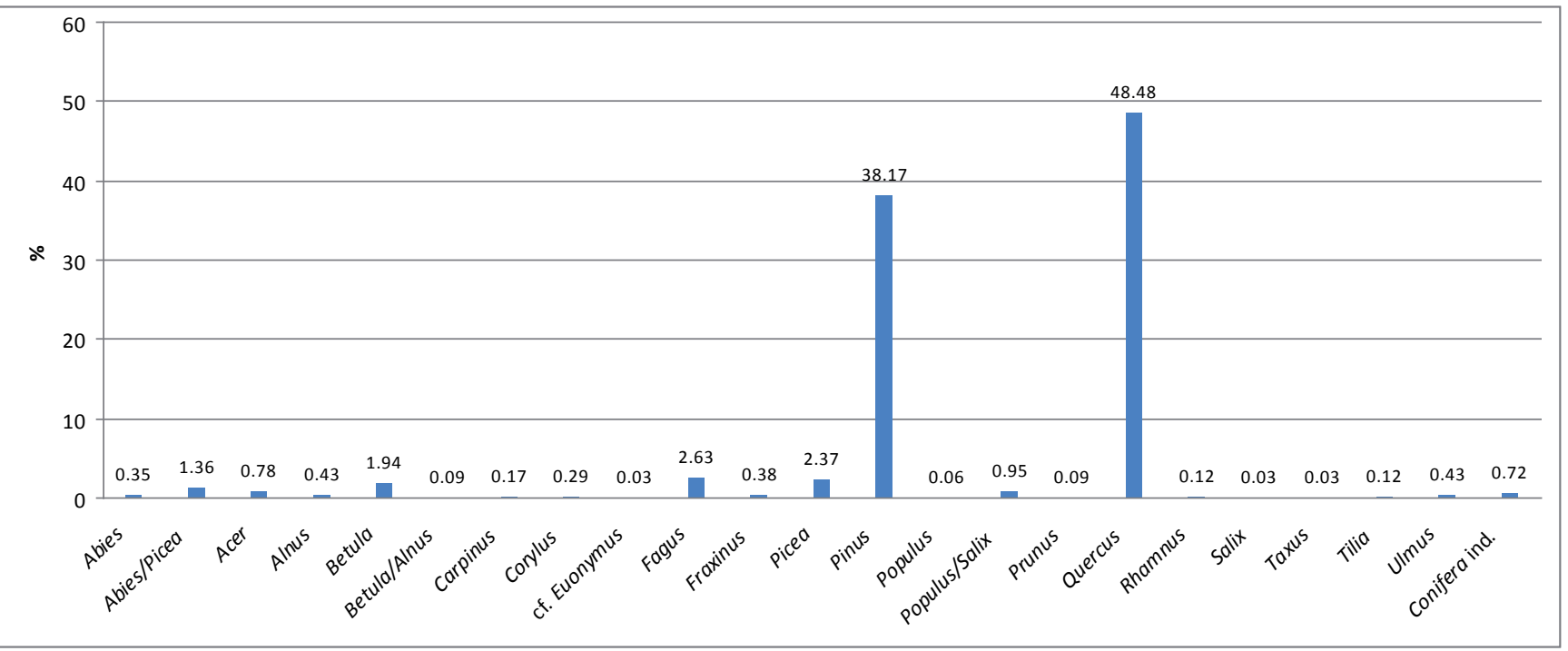

Figure 11. Early Medieval period Žatec - result of charcoal analysis, n=3,461. 
Figure 12. Žatec - Results of charcoal analyses, proportion of groups of local and imported tree taxa, absolute charcoal frequencies $\mathrm{n}=3,461$. "Local" tree taxa: maple (Acer), alder (Alnus), birch (Betula), birch /alder (Alnus/Betula), hornbeam (Carpinus), hazel (Corylus), spindle-tree? (cf. Euonymus), ash (Fraxinus), pine (Pinus), poplar (Populus), poplar /willow (Populus/ Salix), prunus (Prunus), oak (Quercus), buckthorn (Rhamnus), willow (Salix), lime tree (Tilia), elm (Ulmus), undefined conifer - probably pine (Conifera ind.). "Imported tree taxa" - fir (Abies), fir/spruce (Abies/ Picea), spruce (Picea), yew-tree (Taxus), beech (Fagus).

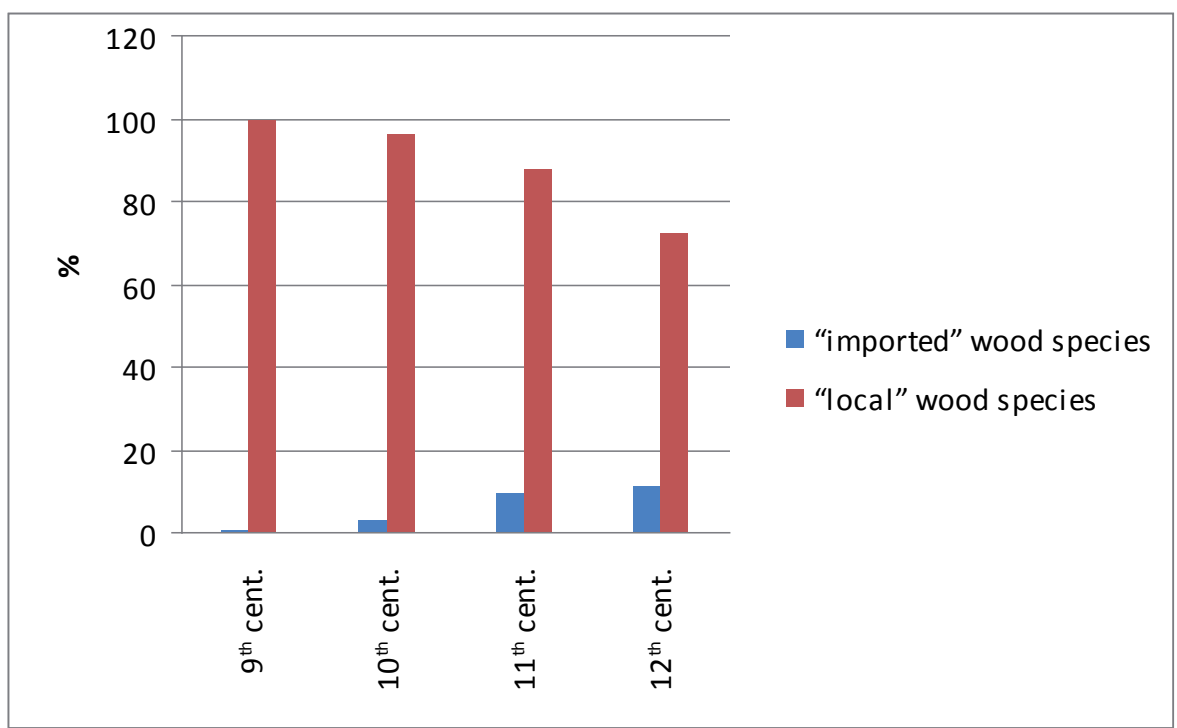

analysed charcoals (from approx. $0.5 \%$ up to $11 \%$ ). In our opinion these results indicate the increasing importance of wood imported from higher altitudes (e.g. the higher slopes of nearby Doupov Mountains). The higher demand for firewood and charcoal that may be associated with the development of iron-working in the $3^{\text {rd }}$ and $4^{\text {th }}$ phases at Žatec might have been satisfied by importing wood from highland localities of fir-beech and beech forests. Transport might have been accomplished by river-rafting along the Ohře River even in the Early Medieval period, although written documents indicate ownership of Př́vlaky village by the Doksy monastery and related river-rafting only in the $1^{\text {st }}$ half of the $13^{\text {th }}$ century (Žemlička 2002).

\section{Conclusion}

The aim of this project was to study the economy of the early medieval Žatec on the basis of archaeobotanical material. The main focus was on the range of cultivated plants, especially macroremains of cereal crops in waste layers associated with consumption. We assume that the proportions of crops in the macroremain assemblage directly reflect the mix of crops produced at the time and their respective importance to the community. Another important focus of the project was on the environmental conditions at Žatec and the region: investigated by means of pollen and macroremain spectra of ruderal plants and firewood charcoal. Additional pollen analysis of putative goat excrement gave us rather unique and detailed information about the urban environment.

For macroremains analysis a total of 101 anthropogenic sediment samples were analyzed from the $9^{\text {th }}$ to $12^{\text {th }}$ centuries. These were largely derived from sunken settlement objects and moats in which plant macroremains remained preserved due to the higher humidity. A total of 15,042 plant macroremains were identified. Of 140 plant species identified, 42 (30\%) were cultivated plants, and of these 20 were domesticated. Based on archaeological data, the time period was divided into four chronological phases, i.e. the $9^{\text {th }}, 10^{\text {th }}, 11^{\text {th }}$ centuries plus the $12^{\text {th }}$ up to the beginning of the $13^{\text {th }}$ century (Čech 2008a).

The archaeological evidence demonstrates two important periods of change. The first is between the $9^{\text {th }}$ and $10^{\text {th }}$ centuries when the structure of the fortified settlement on the promontory was modified. In the $10^{\text {th }}$ century, the entire promontory was fortified. A number of important changes from an archaeological point-of-view occur in the $11^{\text {th }}$ century. At that time, a manor-house with a church was built inside the castle; churches with burial sites and numerous settlements are found surrounding the fortified elements; and there were large centres of iron production and processing in the settlement around the castle. The inventory of materials discovered includes coins -evidencing exchange, glass, and numerous iron and bronze objects. A greater number of churches and development leading up to latest phases of a medieval town are characteristic of the $12^{\text {th }}$ century.

The $9^{\text {th }}$ and $10^{\text {th }}$ centuries are considered the culmination of the oldest phases of the Early Medieval period. The range of cereal crops dominated by naked wheat - both club and common - support this hypothesis. The $11^{\text {th }}$ century brings completely new qualities in all aspects, including archaeobotanical. A sharp increase of rye is documented; however, its proportion decreases in the following centuries, giving way to increases of barley and oats.

The geomorphology of Žatec and the distance from available arable land determine the population density which this land could have supported. In older phases, when 1/5 $1 / 3$ of available land was tilled; a long-term two-field rotation is likely (Beranová 1980, 252). Wheats were the dominant crops during this period. Although a so-called "short- term two-field rotation" is sometimes mentioned, the agricultural system probably remained unchanged until the $12^{\text {th }}$ century (Beranová, Lutovský 2008, 327-328).

The differences among the proportions of cereals (Figure 4) and the relation of cereal crops to summer and winter weeds are evidence of the transformation of the agricultural system to what we believe was a non-regulated three-field (or three- 
course) rotation. The assortment of cereal crops and weeds seems to correlate well with this system. Using winter rye, which is more resistant even if of lower yield, is expected to have stabilized agriculture against climate fluctuations. Nevertheless, a greater area of arable land on which the summer and winter crops could be cultivated successively would have been necessary. Finally higher and more stable yields connected with higher labour inputs would have been achieved.

Changes in the economy of the early medieval Czech state were necessary after a crisis at the end of the $10^{\text {th }}$ century (Sláma 2000, 261-266), when part of the land to the east was lost and the ruling Přemyslids had to find other sources. This was reflected in the beginnings of a foreign policy when Břetislav I (1034-1055) organized a military expedition to Poland (1039). Such an expedition was possible only after stabilization and consolidation of the state economy. We believe that the dating and character of agricultural changes in the economy of Žatec reflect these events.

Plant macroremains, pollen and anthracological analyses have contributed to knowledge of the ecology in and around Žatec. The results demonstrate extensively deforested landscape at least from the $9^{\text {th }}$ century (oldest samples), which is evident from both pollen and charcoals. Pine and oak were the dominant tree species, and grew on soils such as acid sand subsoils that were not attractive for agriculture. The pollen analysis of the animal excrement (most probably of a goat) from the $12^{\text {th }}$ century showed that the animal grazed ruderal vegetation that was available within the town. The animal also probably received branches of hazel and beech.

The data and interpretation presented here are at present only valid for the Žatec complex, since we lack data of comparable detail in both scale and time for comparisons with other sites, in the Czech Republic or abroad. Samples were systematically taken, for example, in the surroundings of the Lesser Town (Malá Strana) at Prague castle, but without further funding these data cannot be processed on a large scale. Currently, the data from Žatec indicate that in the $11^{\text {th }}$ century, an agricultural system already existed that, traditionally, is not expected to appear until the transformations that occurred later in the $13^{\text {th }}$ century.

\section{Acknowledgement}

The article is a component of grant No. IAA800020706 from the Grant Agency of the Czech Academy of Science.

\section{References}

ANDERBERG, A.-L. 1991: Atlas of seeds and small fruits of NorthwestEuropean plant species with morphological descriptions. Part $4-$ Resedaceae - Umbelliferae. Swedish Museum of Natural History, Stockholm, 281.

AKERET, Ö, HAAS, J. N., LEUZINGER, U., JACOMET, S. 1999: Plant macrofossils and pollen in goat/sheep faeces from the Neolithic lake-shore settlement Arbon Bleiche 3, Switzerland. The Holocene 9 (2), 175-182.
BEIJERINCK, W. 1947: Zadenatlas der Nederlandsche Flora ten behoeve ean de Botanie, Paleontologie, Bodemcultuur en Warenkennis. H. Veenman and Zonen, Wageningen, 360.

BERANOVÁ, M. 1975: Zemédélská výroba v 11.-14. století na území Československa. Praha.

BERANOVÁ, M. 1980: Zemědělství starých Slovanů. Praha.

BERANOVÁ, M., LUTOVSKÝ, M. 2008: Slované v Čechách (Archeologie 6.-12. stoleti). Praha.

BERGGREN, G. 1969: Atlas of seeds and small fruits of NorthwestEuropean plant species with morphological descriptions. Part 2 Cyperaceae. Swedish Natural Science Research Council, Stockholm.

BERGGREN, G. 1981: Atlas of seeds and small fruits of Northwest-European plant species with morphological descriptions. Part 3 - Salicaceae Cruciferae. Swedish Museum of Natural History, Stockholm.

BERTSCH, K. 1941: Handbücher der praktischen Vorgeschichtsforschubg: Früchte und Samen. Ein Bestimmungsbuch zur Pflanzenkunde der vorgeschichtlichen Zeit. Ferdinand Enke Verlag, Stuttgart.

BEUG, H. J. 2004: Leitfaden der Pollenbestimmung für Mitteleuropa und angrenzende Gebiete. München.

ČECH, P. 2008a: Současný stav poznání Žatce v raném středověku, Archeologické rozhledy 60, 36-60.

C̆ECH, P. 2008b: Frühmittelalterliche pyrotechnische Produktionsanlagen im Suburbium der Agglomeration von Žatec (Saaz) und die Chronologie der jung- und spätburgwallzeitlichen Keramik. In: Boháčová, I., Poláček, L. (Eds.): Burg - Vorburg - Suburbium. Zur Problematik der Nebenareale frühmittelalterlichen Zentren, Internationale Tagungen in Mikulčice VII. 91-102.

GRAUS, F. 1957: Dějiny venkovského lidu v Čechách v době předhusitské II. Déjiny venkovského lidu od poloviny 13. století do roku 1419. Praha.

JANKOVSKÁ, V. 1997: Výsledky pylových analýz z lokality: Praha 1 Malá Strana, Tržiště 259/III/. In: Kubková, J., Klápště, J., Ježek, M.., Meduna, P. (Eds.): Život v archeologii středověku. Archeologický ústav AV ČR Praha, 299-308.

JANKOVSKÁ, V. 1998: Mostecká cesta, Praha 1-Malá Strana: interpretace pyloanalytického výzkumu.. Pollen Analysis Research Explanation. Final Report. Deposited at. PÚPP, Prague.

JONES, M. K., 1991: Sampling in Palaeoethnobotany. In: Van Zeist W. et al.: Progress in Old World Palaeoethnobotany. Balkema, Rotterdam, 53-63.

KATZ, N. J., KATZ, S. V., KIPIANI, M. G. 1965: Atlas and keys of fruits and seeds occuring in the quaternary deposits of the USSR. Nauka, Academy of Science of the USSR, Commission for the investigation of the quaternary period, Moscow.

KOZÁKOVÁ, R., BOHÁČOVÁ, I. 2008: Přírodní prostředí Pražského hradu a jeho zázemí v raném středověku - výpověd' pylové analýzy sedimentů ze III. nádvoří. Archeologické rozhledy 60, 547-564.

KOZÁKOVÁ, R., POKORNÝ, P. 2007: Dynamics of the biotopes at the edge of a medieval town: pollen analysis of Vltava river sediments in Prague, Czech Republic. Preslia 79, 257-281.

MÁLEK, J. 1983: Problematika ekologie jedle bělokoré a jejiho odumirání. Studie ČSAV 11, Praha.

PEARSALL, D. M. 1989: Palaeoethnobotany: a handbook of procedures. Academic Press, San Diego.

PUNT, W. 1980: The Northwest European Pollen Flora, 37. Umbelliferae. Utrecht.

RASMUSSEN, P. 1993: Analysis of goat/sheep faeces from Egolzwil 3, Switzerland: Evidence for branch and twig foddering of livestock in the Neolithic. Journal of Archaeological Science 20, 479-502.

REILlE, M. 1992: Pollen et spores d'Europe et d'Afrique du nord. Marseille.

SCHERMANN, S. 1967: Magismeret II., Akadémiai kiadó. Budapest. 208. SKÁMA, J. 2000: Ekonomické proměny v přemyslovském státě za panování nástupců Boleslava II. In: Polanský, L., Sláma, J., Třeštík, D. (Eds.), Přemyslovský stát kolem roku 1000, 261-266. Polanský, L., Sláma, J., Třeštík, D. (Eds.): Přemyslovský stat kolem roku 1000. 261-266.

Van der VEEN, M. M. 1984: Sampling for Seeds In: Van Zeist W., Caspaire W. A. (Eds.): Plants and Ancient Man. Balkema. Rotterdam, 193-199.

ŽEMLIČKA, J. 2002: Počátky Čech královských. Praha. 